\title{
Response to the letter to the editor by Nagai $M$ et al. entitled "Right insular cortex atrophy in Takotsubo syndrome: a possible pathogenesis of increased sympathetic nervous system activity?"
}

\author{
Fabian Barbieri $^{1} \cdot$ Noora Tuovinen $^{1} \cdot$ Ruth Steiger $^{2,3} \cdot$ Wolfgang Dichtl $^{1}$ (i)
}

Received: 4 May 2020 / Accepted: 5 May 2020 / Published online: 3 June 2020

(c) Springer-Verlag GmbH Germany, part of Springer Nature 2020

Sirs:

We thank Nagai M et al. for their interest in our study supporting the involvement of frontal brain regions and the central autonomic network in the acute phase of Takotsubo syndrome (TTS). As nicely depicted by their figure, we fully agree that two anatomic aspects of the central nervous system should be emphasized: (1) the specific role of hemispheric laterality, e.g. the different impact of the right and left insular cortex on sympathetic and parasympathetic activities; (2) not only cortical areas, but also several other distinct brain regions may be involved in TTS, including subcortical structures, the brain stem and the spinal cord.

In our study, no significant functional connectivity differences between amygdala and insula were detected in TTS patients compared to healthy controls. The brain atlas that we used includes separate regions for the left and right amygdala and subregions for the left and right insula. We have not found any significant differences between TTS patients and healthy controls when we assessed separately the within-network connectivity of different resting-state networks including the salience network. It is true that we have not looked so far into the sympatho-vagal balance relation to connectivity, which is especially interesting

This reply refers to the comment available online at https://doi. org/10.1007/s00392-020-01665-2.

Wolfgang Dichtl

wolfgang.dichtl@i-med.ac.at

1 University Hospital for Internal Medicine III (Cardiology and Angiology), Medical University of Innsbruck, Innsbruck, Austria

2 Department of Neuroradiology, Medical University of Innsbruck, Innsbruck, Austria

3 Neuroimaging Research Core Facility, Medical University of Innsbruck, Innsbruck, Austria concerning the insular cortex (e.g. by additional correlation analysis of heart rate parameters on connectivity).

Given the complexity of the central nervous system and the limitations of our currently available research tools (despite great advances such as functional MRI), we are not fully convinced that the underlying mechanism for the presented data can be so easily provided as claimed by the authors. In contrast, we feel the risk of inappropriate simplifications and prefer to approach this new exiting experimental field with some reluctance and humility.

The scientific journey of understanding the heart-brain axis has just begun, and TTS might function as a disease model, given the high incidence of neurological and/or psychiatric comorbidities. TTS is not only damaging the heart, but also it is much more than a cardiomyopathy. It is a complex entity, in many cases involving the central nervous system, targeting several organs and cell types. This paradigm shift has to be incorporated more into our clinical work and research. In consequence, we suggest performing more extracardiac imaging in TTS patients, in collaboration with experts from other disciplines including neuroscience.

\section{Conflict of interest}

The authors declare no conflict of interest. 